\title{
Mesure et représentation cartographique de l'impression de confinement d'un piéton immergé dans la ville
}

\author{
Houda Belgacem ${ }^{1,3}$, Thomas Leduc ${ }^{1,3}$, et Marjorie Musy ${ }^{2,3}$ \\ ${ }^{1}$ UMR AAU/CRENAU, CNRS, Nantes, France \\ ${ }^{2}$ Cerema, Nantes, France \\ ${ }^{3}$ IRSTV - FR CNRS 2488 - Nantes, France
}

\begin{abstract}
Résumé. Nous présentons dans cet article un essai de quantification de l'impression de confinement d'un piéton immergé dans la ville. Cette quantification repose sur un ensemble de qualités perceptuelles évoquées par (Ewing et al., 2006) ou (Dupagne et al., 1999) telles la mesure de l' « enfermement urbain », la « cohérence formelle », la « profondeur urbaine » ou le « rapport d'échelle ». Nous y associons des indicateurs provenant de la littérature scientifique comme le facteur de vue du ciel et la longueur de vue maximale et proposons de nouveaux indicateurs tels un ratio d'aspect moyen de l'espace ouvert immédiatement environnant, une hauteur moyenne du déroulé de façades environnant, une complexité formelle et un angle solide de vision (une déclinaison de la carte de vue du ciel pour une ouverture d'angle verticale préfixée). La cartographie qui résulte de cette quantification met en évidence une grande variété d'espaces.
\end{abstract}

Mots-clés. Perception visuelle, ouverture, perméabilité, confinement, vide urbain, SVF.

\begin{abstract}
In this article, we present an attempt to quantify the impression of confinement of a pedestrian immersed in the city. This quantification is based on a set of perceptual qualities induced by (Ewing et al., 2006) or (Dupagne et al., 1999), like the measures of "urban confinement", "coherence", "urban depth" or the "human scale". We combine indicators from the scientific literature, such as the sky view factor and the maximum view length, and propose new indicators like an average aspect ratio of the immediately surrounding open space, an average height of the surrounding façades, a geometrical complexity and a solid angle of vision (a variation of the sky map for a prefixed vertical angle aperture). The mapping that results from this quantification highlights a wide variety of spaces.
\end{abstract}

Keywords. Visual perception, Aperture, Permeability, Confinement, Urban void, SVF. 


\section{Introduction}

Dans sa communication de 2006, dans le colloque intitulé « la densité peut-elle enrayer l'étalement urbain », Marc Wiel met en évidence la polysémie de la densité (Wiel, 2006). Il « rappelle que le sentiment d'entassement n'est pas proportionnel à la densité objective d'occupation du sol », évoque une densité « vécue » ou facette «psychosociologique »du terme, qu'il complète d'une dimension « économique ». L'auteur suggère également que la densité pourrait mesurer « une sorte de pression urbaine » et qu'elle serait assimilable à un processus («Elle ne mesure plus seulement un niveau instantané d'entassement ou de pression urbaine en un lieu, elle est un repère dans une évolution continue du profil des densités » (ibid.)). Weil complète son propos en arguant "que la façon d'agréger et de disposer les diverses formes d'occupation du sol permet d'obtenir une bonne rentabilisation de l'occupation du sol sans donner le sentiment d'entassement, au contraire en donnant un surcroît de satisfaction ». Il n'est pas le seul auteur à distinguer une densité objective, supervisée, extérieure, comme en surplomb, d'une densité vécue, ressentie, en immersion dans la ville. L'Atelier d'Urbanisme Parisien dans son rapport sur les densités vécues appliquées à quatre quartiers parisiens (APUR, 2003), met ainsi en évidence qu'une forme dense et compacte n'est pas antinomique d'une satisfaction résidentielle. «A l'opposé, le quartier Falguière-15e, le moins densément bâti des quatre est celui avec Jeanne d'Arc-13e, où les habitants estiment majoritairement qu'il y a trop de constructions. L'idée d'une influence de la forme urbaine sur la perception de la densité semble trouver ici des arguments » (ibid., p. 64). La configuration spatiale semble « déterminante puisque le nombre même de logements à l'adresse de l'enquêté est corrélé au sentiment d'entassement » (ibid., p. 63).

Par ailleurs, (Takei et al., 1978) ont montré que la configuration d'une zone urbaine est le facteur le plus influent sur le sentiment d'oppression. Les dimensions plastiques et l'esthétique des lieux, leur accessibilité, l'agencement des bâtiments et leur nombre constituent un ensemble « de signaux sensoriels » captés par l'individu (APUR, 2003). Ils renvoient un vécu positif ou négatif, un sentiment de sécurité ou de crainte, de confinement ou de promiscuité. (Leroux et al., 2013, p. 108) évoquent des phénomènes de compression ou de propulsion pour décrire l'espace enveloppant par articulation entre les espaces bâtis et non bâtis. La compression ou rétrécissement de l'espace vécu est associée à un sentiment de repli sur soi tandis que la propulsion provoque au contraire un sentiment de dilatation, projection, impulsion à aller de l'avant. (Ewing et al., 2006) proposent une liste de qualités perceptuelles susceptibles d'être utilisées pour la qualification d'un milieu urbain en relation à cette notion de densité ressentie. Nous les présentons ici comme des variables et distinguons enclosure, human scale et coherence qui semblent plus immédiatement liées au sentiment de confinement. (Ewing et al., 2006) évoquent d'autres variables de mesure de l'environnement à l'échelle de la rue telles que imageability, legibility ou linkage. Ces dernières sont plus liées à la création d'une carte mentale chez le piéton et servent à l'orientation ou à la description des milieux déjà traversés. Transparency fait aussi partie de la liste et est associable au sentiment de confinement, mais pour des raisons de granularité des données à disposition (utilisation des référentiels géographiques nationaux dépourvus de toute référence à la texture des bâtiments), nous l'éliminons de notre sélection.

Dans le cadre de cette recherche, notre objectif est de proposer et de mettre en œuvre une implémentation de trois variables choisies par (Ewing et al., 2006) auxquelles nous ajoutons une quatrième évoquée par (Dupagne and Teller, 1999). Notre but est de rendre compte de l'expérience corporelle du piéton se déplaçant dans un environnement urbain. Pour ce faire nous exploitons des indicateurs à portée locale (tableau 1), en fonction des positions successives du piéton. Dans la partie méthodologique, nous commençons par présenter le principe de chaque indicateur et son implémentation. Nous appliquons ensuite 
nos calculs d'indicateurs sur un cas d'étude urbain à Nantes. Les résultats sont analysés dans la dernière partie.

Tableau 1. Présentation des 4 variables et des 6 indicateurs associés.

\begin{tabular}{|c|l|}
\hline Variables urbaines & \multicolumn{1}{|c|}{ Indicateurs associés } \\
\hline Enclosure (Enfermement) & $\begin{array}{l}\text { 1/ Ouverture de ciel (SVF) (Oke 1988) } \\
\text { décrit l'occlusion urbaine. } \\
\text { 2/ Ratio d'aspect moyen : généralisation de } \\
\text { 1'indicateur habituellement calculé dans le } \\
\text { cadre d'un modèle de rue canyon (hauteur } \\
\text { du bâti rapporté à la largeur de la voie } \\
\text { correspondante) }\end{array}$ \\
\hline Profondeur & 3/ Percée visuelle ou plus grande longueur \\
& de vue (Dupagne and Teller, 1999) \\
\hline Cohérence & 4/ Mesure de la circularité de l'isovist : \\
& l'isovist est un polygone du plan horizontal \\
& composé de l'ensemble des points visibles \\
& dans toutes les directions de l'espace \\
& depuis une position donnée (Stamps III, \\
\hline Human Scale (Rapport & 2005) \\
d'échelle) & 5/ Angle solide de vision \\
& 6/ Hauteur moyenne des bâtiments \\
\hline
\end{tabular}

\section{Méthodologie et implémentation}

Dans le tableau 2, nous exposons la méthode de calcul pour chaque indicateur. Tous les indicateurs caractérisent le paysage urbain dans une approche panoptique (traitement homogène dans toutes les directions d'un plan horizontal, sur $360^{\circ}$ ). Ils reposent tous, en termes d'implémentation informatique, sur le mécanisme du lancer de rayons (figure 1). Les formules présentées dans le tableau 2 sont adaptées pour être utilisées dans le contexte d'un SIG 2D (avec des jeux de données vectorielles classiques telles celles issues des référentiels géographiques nationaux).

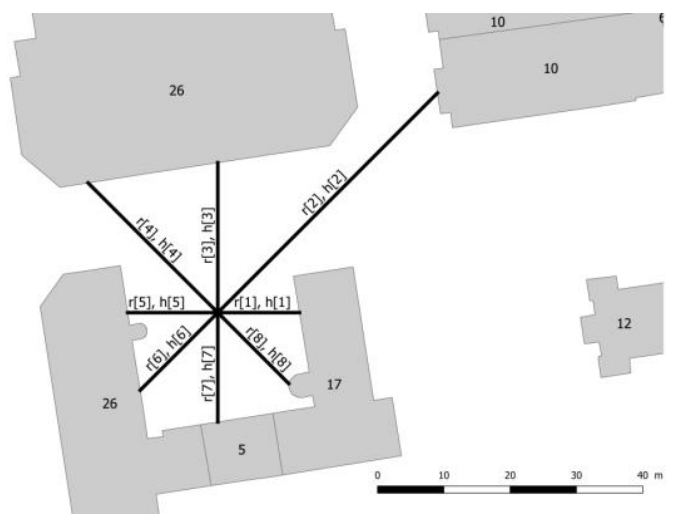

Figure 1. Ensemble de rayons lancés à partir d'une position donnée avec les attributs $r_{k}$ (longueur de rayon) et $\theta_{\mathrm{k}}$ (azimut) correspondants. Les élévations des bâtiments sont inscrites dans les empreintes de bâtiment correspondant. 


\subsection{Enfermement urbain : SVF et ratio d'aspect moyen}

Le SVF correspond au pourcentage de ciel vu (c'est-à-dire à la portion de ciel qui est visible à partir du point de vue, au sens où il n'est pas obstrué par un masque bâti environnant, figure 2). Il est étroitement lié à la largeur de l'espace ouvert et aux hauteurs et largeurs des diverses obstructions (figure 3). Dans notre étude de cas, l'obstruction se limite aux seuls bâtiments et n'intègre par exemple ni la végétation ni le mobilier urbain.

Pour compléter ce ratio de ciel vu et en référence aux travaux de (Stamps III, 2005) qui propose un raffinement de la définition de l'enfermement par des formules mathématiques liées aux propriétés de l'isovist (élongation, surface, concavité, etc.), nous proposons d'implémenter une généralisation du ratio d'aspect ou ratio d'aspect moyen. Le ratio d'aspect est un indicateur qui, dans l'état de l'art, s'applique aux seules rues canyons (Oke, 1988). Il consiste en un rapport de hauteur des bâtiments environnants rapportée à la largeur de la voie. Notre généralisation consiste en une version panoptique de ce rapport de hauteur sur largeur (figure 4). Dans cette version et, par application du lancer de rayons (figure 1), nous rapportons chaque hauteur de bâtiment interceptant un rayon par la longueur de ce même rayon avant de calculer la moyenne des rapports (figure 5).

\subsection{Profondeur : percée visuelle ou plus grande longueur de vue}

On appelle longueur de vue (dans une direction donnée), la distance qui sépare l'observateur de la première face de bâtiment rencontrée dans cette même direction. Dans le cadre de notre étude nous nous intéressons à la plus grande longueur de vue associée à une position de l'observateur. En effet, d'après (Natapov et al., 2016), au cours de leurs trajets, les piétons choisissent, les routes les plus droites possible (profondeur du champ de vision longue) par opposition aux routes les plus sinueuses disponibles (profondeur du champ de vision courte). Dans notre étude, nous nous focalisons sur la profondeur maximale du champ de vision (figure 6) et, pour ce faire, nous cherchons le maximum des longueurs des rayons à l'aide de l'algorithme de lancer de rayons.

\subsection{Cohérence urbaine : circularité de l'isovist}

Nous cherchons ici à qualifier le «désordre formel urbain» lié à l'arrangement des bâtiments. Pour mesurer cette grandeur, nous identifions un ensemble de points de l'espace ouvert puis, en chacun de ces points, nous calculons l'isovist correspondant et l'écart type des longueurs des rayons associés. Plus l'écart type est élevé, plus le contour de la zone est complexe (figure 7) rendant ainsi l'environnement moins cohérent en apparence.

\section{4 Échelle humaine : angle solide de vision}

Les indicateurs que nous avons présentés sont nos outils pour décrire la relation entre l'espace construit et l'espace non construit que nous appelons aussi espace ouvert. En vue de profil, et en focalisant sur la section de la rue, le SVF et le ratio d'aspect informent sur la géométrie de vide interstitiel. La distance de visibilité maximale définit l'étendue de l'espace vide rapportée au point de vue correspondant. Et finalement la cohérence détermine le degré d'imbrication du bâti par rapport au non bâti. 
Tableau 2. Présentation des six indicateurs mis en œuvre dans le cadre de notre étude. Tous quantifient le vide urbain.

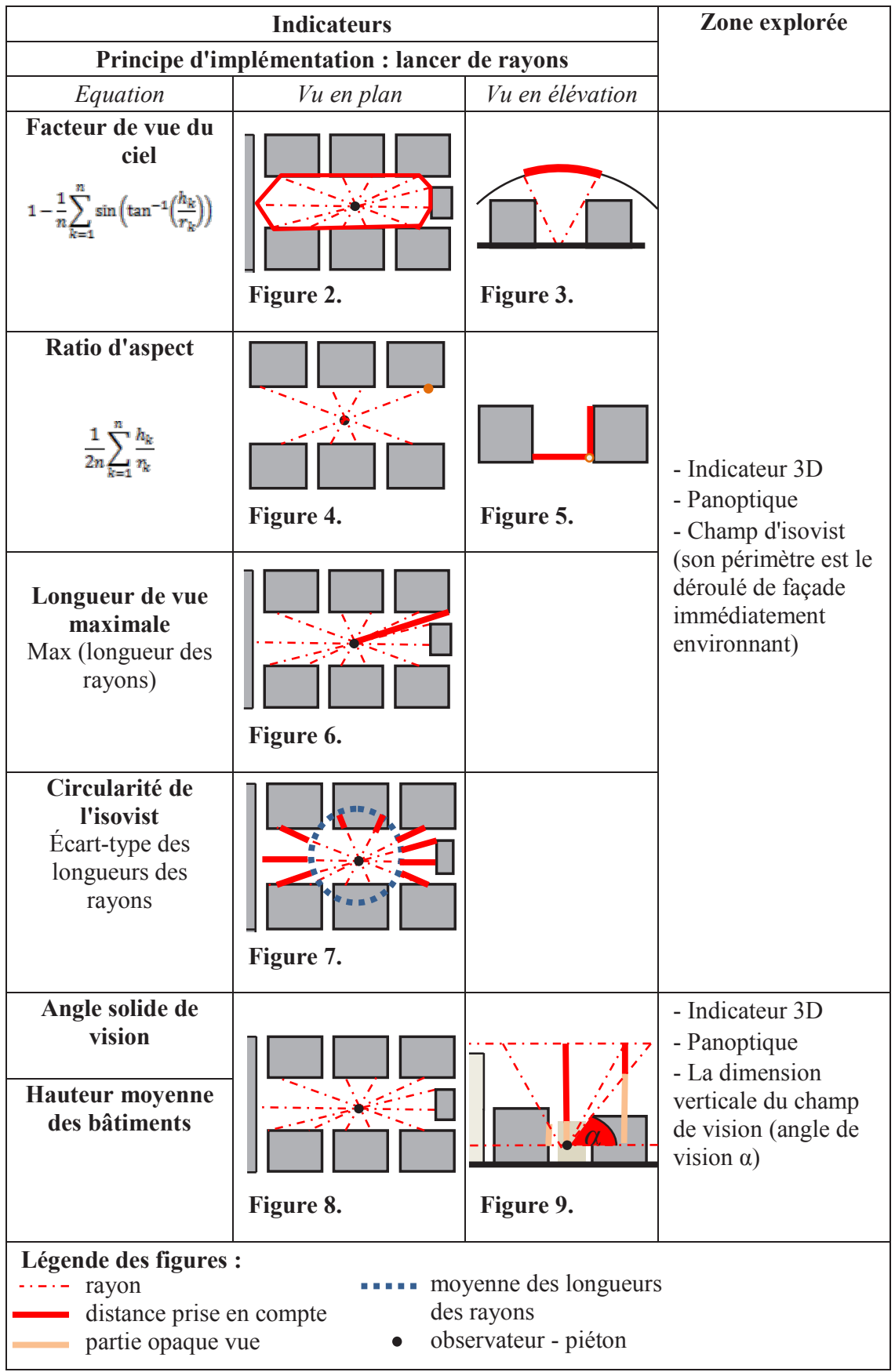

Pour approcher au mieux la perception visuelle du piéton, on ajoute un cinquième indicateur, appelé « angle solide de vision ». L'objectif est ici d'évaluer le ratio de vide et de plein pour un secteur angulaire (vertical) donné (une ouverture égale à l'angle visuel de $30^{\circ}$ (Robert, 2013) (figure 9)), et pour une ouverture horizontale panoptique $\left(360^{\circ}\right.$, 
figure 8). Notre choix d'étudier le secteur de l'angle solide de vision est lié aux travaux de (Asgarzadeh et al., 2012) qui l'exploite comme outil d'analyse pour mesurer le sentiment d'oppression d'un sujet exposé à un environnement urbain dense. Dans notre étude, cet indicateur sert à évaluer le degré de perméabilité visuelle du milieu. Le caractère panoptique de cet indicateur le démarque de l'étude de (Robert, 2013) qui fixe l'ouverture du champ de vision à $150^{\circ}$. Comme nous n'analysons pas des trajets mais plutôt un ensemble de positions hors de toute considération d'orientation, toutes les directions du champ de vision sont prises en compte de manière équivalente. Notre sixième et dernier indicateur procède de la même manière et s'attache à mesurer la hauteur moyenne des bâtiments dont les façades bornent le champ de vision du piéton

\section{Résultats et discussion}

On a choisi comme cas d'étude une rue piétonne dans le quartier Bouffay au centre de la ville de Nantes. L'intérêt est de pouvoir traiter la densité sensible sur toute la surface intercalée entre les bâtiments (cela traduit l'emplacement du piéton près du bâtiment, un peu loin et du côté opposé de la rue). Pour ce faire, on a visé une rue piétonne qui est connue pour une fréquentation importante par la population. Les positions depuis lesquelles on a lancé le calcul de la liste des indicateurs traduisent en quelque sorte les positions probables du piéton lors de son passage. Elles sont placées selon une grille de dimensions de $1 \mathrm{x} 1 \mathrm{~m}$.

Les figures 10a (ratio d'aspect moyen) et 10b (SVF) permettent de constater que ces deux indicateurs sont globalement inversement proportionnels. Plus le ratio d'aspect est grand, plus les masques bâtis sont élevés et l'espace ouvert resserré et plus le ciel est masqué (i.e. plus le SVF tend vers 0 ). Ce ratio est ainsi plus faible aux intersections, endroits où la pression de l'environnement construit se « relâche ».
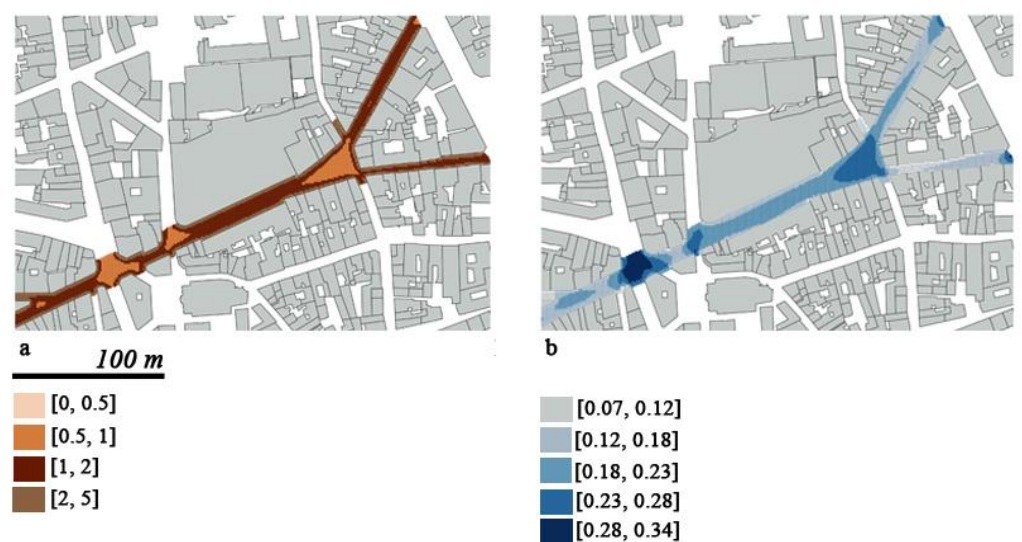

Figure 10. a. Variation du ratio d'aspect moyen, b. Variation du SVF.

Sur la figure 11a on note que les valeurs de l'indicateur de l'angle solide de vision augmentent aux intersections. Les croisements des rues et les places avec des échappées visuelles coïncident aussi avec des valeurs importantes. Par contre dans les impasses où le milieu est étroit, l'angle solide de vision est faible. Cet indicateur est aussi faible à proximité des enveloppes de bâtiments. 

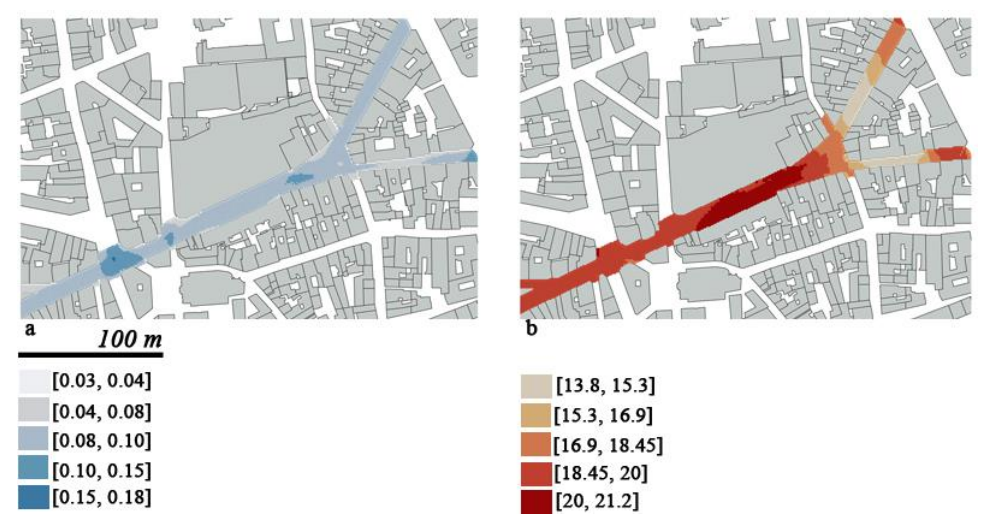

Figure 11. a. Variation de l'angle solide de vision, b. Variation de la hauteur moyenne.

Le SVF et le ratio d'aspect panoptique $\mathrm{H} / \mathrm{W}$ présentent une relation de proportionnalité (figure 10), l'un est donc réductible à l'autre. Cette corrélation justifie que l'on ne retienne, pour la suite de ce document, que le SVF. On a éliminé aussi le paramètre de la cohérence urbaine pour se concentrer sur le pourcentage du vide par rapport au plein et sur la profondeur de vision. Par une approche empirique, nous choisissons de combiner les quatre indicateurs que sont l'angle solide de vision, la hauteur moyenne, la distance de visibilité maximale et le SVF (équation 1). La carte de la figure 12 est le produit de cette démarche empirique qui vise à représenter la sensation de confinement du piéton en milieu urbain. L'indicateur composite associé est formulé de la manière suivante :

\section{$(1 / 4) *($ Angle solide de vision + (Hauteur moyenne/maximum(Hauteur moyenne)) + (Longueur de vue maximale/(maximum(Longueur de vue maximale))) + SVF) (éq. 1)}

Sur la figure 12 les intervalles des valeurs varient entre 0 et 0.60 . Les valeurs sont normalisées et permettent de mettre successivement en évidence : entre 0 et 0.40 des espaces clos accompagnés d'une sensation de "resserrement»; entre 0.45 et 0.50 des espaces «modérés" avec une sensation d'équilibre; au-delà de 0.50 des espaces très ouverts accompagnés d'un sentiment d'exposition. Ces seuils restent à valider par un recueil de perceptions situées.

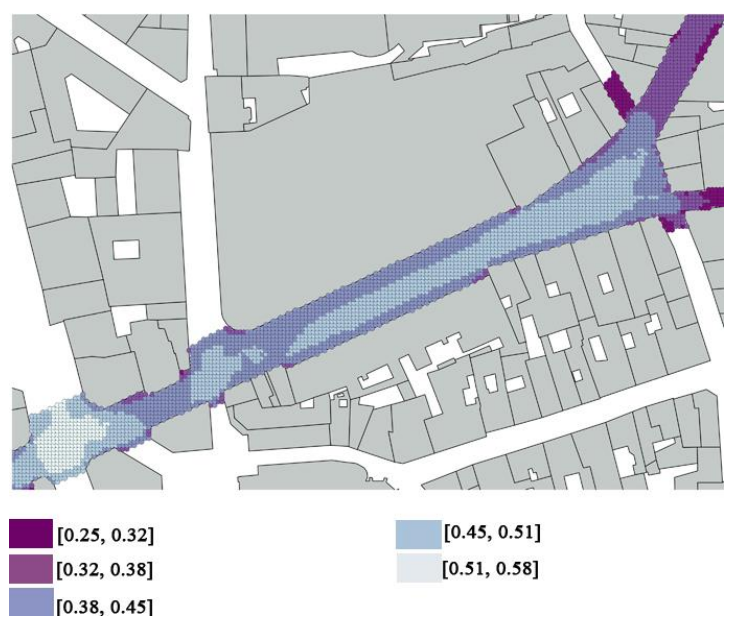

Figure 12. Cartographie de l'indicateur composite. 
Dans le but de rendre la formulation (équation 1) plus simple et de la «consolider », nous avons cherché à réduire le nombre des indicateurs en jeu. Pour commencer, nous avons normalisé tous les paramètres pour harmoniser l'équation, puis, constatant son très faible impact, nous avons éliminé la hauteur moyenne. Restent alors dans l'équation, l'angle solide de vision, le SVF et la longueur maximale, tous normalisés. La figure 13 compare l'angle solide de vision au SVF. Les deux restituent deux informations urbaines différentes, l'angle solide ne présentant en effet qu'une partie de la vue du ciel (celle qui se limite à une bande horizontale adaptée au champ de vision et sur $360^{\circ}$ ). C'est une forme de rétrécissement du SVF à l'échelle de l'observateur.
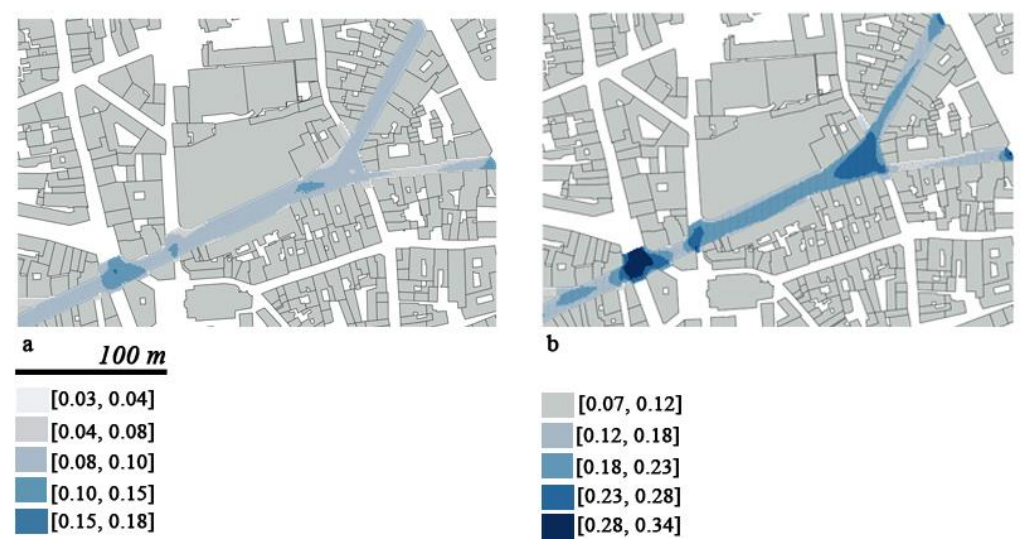

Figure 13. a. Variation de l'angle solide de vision, b. Variation du SVF.

Une dernière modification a consisté en l'élimination complète du SVF pour ne garder que l'angle solide et éviter ainsi la redondance d'information. Les résultats correspondants sont cartographiés sur la figure 14 .

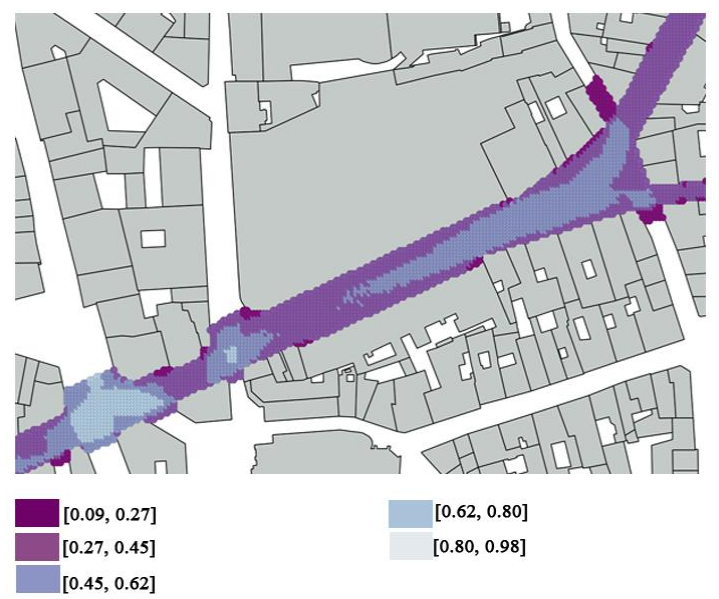

Figure 14. Cartographie de l'indicateur composite sur la zone d'étude sélectionnée.

La figure 14 montre un résultat semblable à celui de la figure 13. Les limites des intervalles sont différentes, mais les valeurs seuils déduites dans le paragraphe précédent restent à peu près semblables. Notre indicateur composite de confinement peut ainsi être 
simplifié pour ne garder que deux paramètres : l'angle solide et la longueur maximale (équation 2).

$(1 / 2) *(($ Angle solide de vision / maximum (Angle solide de vision $))+($ Longueur de vue maximale/(maximum(Longueur de vue maximale))))

(équation 2)

\section{Conclusion}

Nous avons proposé dans cet article un nouvel indicateur, appelé «angle solide de vision », qui est une déclinaison du classique facteur de vue du ciel adapté à la perception visuelle d'un citadin en environnement urbain, un ratio d'aspect moyen panoptique et une hauteur moyenne panoptique sur la zone visible par le piéton. En outre, nous avons cherché à cartographier la sensation de confinement. Nous avons ainsi présenté une première étape d'une proposition d'analyse de l'espace pour évaluer l'impression du piéton en réaction à certaines caractéristiques spatiales dans un contexte urbain défini. Le travail sera poursuivi dans le cadre d'un stage par des enquêtes de terrain qui permettront, par des observations in situ, de mesurer la validité de notre proposition. Il s'agira de comparer les résultats aux perceptions réelles et de calibrer les formules empiriques des indicateurs ou d'ajouter d'autres facteurs à l'indicateur composite.

\section{Bibliographie}

APUR. 2003. Densité vécue et formes urbaines, étude de quartiers parisiens.

Asgarzadeh, M., Lusk, A., Koga, T., and Hirate, K. (2012). Measuring Oppressiveness of Streetscapes. Landscape and Urban Planning 107(1) : 1-11.

Dupagne, A., et Teller, J. (1997). Représentation de l'espace ouvert dans un système d'information de projet urbain (version 2).

Ewing, R., Handy, S., Brownson, R., Clemente, O., and Winston, E. (2006. Identifying and Measuring Urban Design Qualities Related to Walkability. Journal of Physical Activity and Health 3(Suppl 1) : 223-40.

Leroux, M., Thibaud, J.-P., Balez, S., Bardyn, J.-L., et Fiori, S. (2000). Composition sensibles de la ville. Ed. Ministère de l'équipement, des transports et du logement, Direction de l'habitat et de la construction, Recherche F97-97.

Natapov, A., et Fisher-Gewirtzman, D. (2016). Visibility of Urban Activities and Pedestrian Routes : An Experiment in a Virtual Environment. Computers, Environment and Urban Systems $58: 60-70$.

Oke, T. R. (1988). Street Design and Urban Canopy Layer Climate. Energy and Buildings $11(1-3): 103-13$.

Robert, J.-M. (2013). Les Angles Visuels. 15.

Stamps III, Arthur E. 2005. Isovists, Enclosure, and Permeability Theory. Environment and Planning B: Planning and Design 32(5):735-62.

Takei, M., et Oohara, M. (1978). Experimental study on measurement of the sense of oppression by a building : (Part 3) consideration of the distance to a building, and relation between color effect of exterior wall and the sense of oppression. Transactions of the Architectural Institute of Japan, (No. 263), 71-80 (Japanese)

Wiel, M. (2006). Polysémie de La Densité: Comment Vivre Avec... in UrbaPlus, Le Réseau de l'Institut d'Urbanisme de Paris. Colloque 2006 - Denses Cités : La densité peut-elle enrayer l'étalement urbain? Paris la Défense, France. 